\title{
TRATAMIENTO DE LAS AGUAS RESIDUALES DEL PROCESO DE CURTIDO TRADICIONAL Y ALTERNATIVO QUE UTILIZA ACOMPLEJANTES DE CROMO
}

\author{
Hansel Miguel Córdova Bravo ${ }^{\mathrm{a}^{*}}$, Rocío Vargas Parker ${ }^{\mathrm{b}}$, Mary Flor Cesare Coral $^{\mathrm{a}}$, \\ Lisveth Flores del Pino ${ }^{a}$, Lizardo Visitación Figueroa ${ }^{a}$
}

\begin{abstract}
RESUMEN
Los métodos tradicionales de producción de cueros en el Perú consumen en promedio entre el 60 al 80\% del cromo utilizado para el proceso de curtido, generando aguas residuales con altas concentraciones de cromo total que deben ser tratadas para poder cumplir con los Valores Máximos Admisible (VMA) establecidos por el DS 029-2009-Vivienda. El uso de agentes acomplejantes de cromo en el proceso de curtido permite reducir la oferta de cromo incrementando la absorción en la piel. El objetivo de la presente investigación fue evaluar la tratabilidad por precipitación química de las aguas residuales de los procesos tradicionales y alternativos de curtido, además de comparar las condiciones de tratamiento de los efluente de curtido de un proceso tradicional, con procesos alternativos que utilizan agentes acomplejantes y basificantes. Se determinó que el $\mathrm{pH}$ óptimo para la precipitación estuvo entre 7,36 y 9,99; además, las dosis utilizadas de hidróxido de sodio estuvieron entre 0,5 y 5,5 $\mathrm{g} / \mathrm{L}$, siendo el proceso tradicional el que requiere mayor dosis. Se determinó que el costo de los insumos empleados en la producción del cuero a wet blue es mayor al costo de insumos empleados en el tratamiento de las aguas residuales de curtido, cuando los valores de cromo total son menores a $650 \mathrm{mg} / \mathrm{L}$.
\end{abstract}

Palabras clave: Precipitación química, curtido, wet blue, cromo

\begin{abstract}
Traditional methods of leather production in Peru consume on average between 60 to $80 \%$ of chromium for tanning process, generating wastewater with high concentrations of total chromium that must be treated in order to meet the Maximum Allowable Values ( VMA ) established by the DS 029-2009 - Vivienda. The use of complexing agents in the chrome tanning process reduces the supply of chromium to increase skin absorption. The main objective of this study was to evaluate the treatability by chemical precipitation of wastewater from traditional and alternative tanning process. It was determined that the optimal $\mathrm{pH}$ for the precipitation was between 7.36 and 9.99, the doses used besides sodium hydroxide were between 0.5 and $5.5 \mathrm{~g} / \mathrm{L}$, is the traditional process that requires higher dose . It was determined that the cost of the inputs used in the production of wet blue leather is more than the cost of inputs used in the treatment of wastewater from tanning when total chromium values are less than $650 \mathrm{mg} / \mathrm{L}$.
\end{abstract}

Key words: Chemical precipitation, tanning, wet blue, chrome

a Centro de Investigación en Química, Toxicología y Biotecnología Ambiental del Departamento Académico de Química de la Facultad de Ciencias de la UNALM Lima - Perú.

e-mail: hansel_cordova@yahoo.com

a Helianthus SAC, Laboratorio de desarrollo e investigación curtiembre, Av. Guardia Civil \#314 Chorrillos - Lima - Perú. 


\section{INTRODUCCIÓN}

El proceso de curtido se resume como las etapas en los cuales la piel de diferente tipos de animales son transformados en cuero. Durante el proceso se diferencian cuatro etapas: $\mathrm{La}$ etapa de ribera, que consiste en la recepción de pieles, salado, remojo y/o lavado, pelambre, descarnado y dividido. La etapa de curtido, que consiste en el desencale y purgado, lavado, piquelado, curtido y basificado; al culminar esta etapa el cuero es llamado wet blue. La etapa de post-curtido, que consiste en el escurrido, rebajado, neutralizado, lavado, recurtido, teñido y engrasado, lavado y secado. Finalmente la etapa de acabado, que consiste en el acondicionado, palizonado y tesado, pintado, batanado y planchado; al culminar esta etapa obtenemos el cuero acabado.

Para el año 2013 se estima que existen 92 fábricas de curtido en el Perú: en Lima (14), Trujillo (48) y Arequipa (30); estas fábricas brindan servicio a 375 empresas que concesionan el procesamiento del cuero: en Lima (50), Arequipa (104) y Trujillo (221) ${ }^{1}$. En el proceso de curtido tradicional la piel consume en promedio $60 \%$ a $80 \%$ del cromo aplicado, el resto es descargado como agua residual industrial, causando problemas al sistema de alcantarillado, incumpliendo los Valores Máximos Admisible (VMA) establecidos por el DS 029-2009Vivienda, impactos ambientales y desventajas económicas para los curtidores ${ }^{2,3,4}$.

Existen muchos métodos para la remoción de cromo total, de todos estos la precipitación química es la más usual ${ }^{2}$. Varios factores afectan el proceso de precipitación, tales como el pH y la formación de complejos ${ }^{5,6,7}$.

El uso de acomplejantes de cromo en el baño de curtido, mejora la tasa de penetración y permite la basificación a $\mathrm{pH}$ 's altos, aumentando su estabilidad frente a álcalis, tamponando la solución de complejos de cromo. El cromo acomplejado penetra mejor en la piel y hace más uniforme la distribución del cromo en su sección transversal ${ }^{8}$. El uso de agentes acomplejantes del cromo disminuye la oferta de cromo en el baño y mejora el proceso de absorción, logrando reducir la concentración de cromo en el efluente final; de esta forma el gasto por tratamiento de este efluente de curtido se reduce?.

El propósito de esta investigación fue evaluar la tratabilidad por precipitación química de las aguas residuales de los procesos tradicionales y alternativos, además de comparar las condiciones de tratamiento de los efluentes de curtido de un proceso tradicional con procesos alternativos que utilizan agentes acomplejantes y basificantes.

\section{Materiales y métodos}

\section{PARTE EXPERIMENTAL}

\section{Procedimientos de curtido}

Se evaluó cinco procesos de curtido: el proceso Tradicional (T) simula las condiciones de curtido promedio de las curtiembres en el Perú; los procesos alternativos AI, AII, AIII y AIV utilizan agentes acomplejantes y basificantes para reducir la oferta de cromo III en el baño de curtido, como se indica en la tabla 1. 
Tabla 1. Materias primas utilizadas en los diferentes procesos de curtido.

\begin{tabular}{lcccccc}
\hline \multicolumn{1}{c}{$\begin{array}{c}\text { Materia } \\
\text { prima }\end{array}$} & Unidad & \multicolumn{5}{c}{ Proceso } \\
\cline { 3 - 7 } & & $\mathrm{T}$ & $\mathrm{AI}$ & AII & AIII & A IV \\
\hline Masa & $\mathrm{kg}$ & 0,24 & 0,22 & 0,002 & 0,010 & 0,003 \\
& & & & & & \\
Agua & $\mathrm{kg}$ & 200,00 & 88,00 & 2,100 & 3,880 & 2,940 \\
Sal & $\mathrm{kg}$ & 17,00 & 8,80 & 0,084 & 0,388 & 0,176 \\
Tanplex FLA & $\mathrm{kg}$ & & 2,20 & 0,021 & 0,097 & 0,029 \\
Ácido fórmico & $\mathrm{kg}$ & 6,50 & 1,10 & 0,017 & 0,029 & 0,015 \\
Tanplex SS & $\mathrm{kg}$ & - & 1,21 & 0,012 & 0,049 & 0,015 \\
Cromo 33 & $\mathrm{kg}$ & 19,20 & 12,10 & 0,116 & 0,486 & 0,148 \\
Bicarbonato & $\mathrm{kg}$ & 3,38 & - & - & - & - \\
de sodio & & & & & & \\
Tanplex CRII & $\mathrm{kg}$ & - & 0,88 & 0,013 & 0,039 & 0,018 \\
Neutran BMR & $\mathrm{kg}$ & - & 0,44 & 0,006 & 0,015 & 0,004 \\
\hline
\end{tabular}

Tanplex FLA: Complejante de calcio libre de nitrógeno. Actúa en el desencale y parte del piquelado. Reemplaza al ácido fórmico.

Tanplex SS: Sales y ácidos orgánicos acomplejante de cromo, favorece la distribución estratificada del cromo y mejora el agotamiento del baño.

Cromo 33: Sal de sulfato de cromo al 25,5\%.

Tanplex CR II: Mezcla sinergética de substancias complejantes de cromo. Mejora la distribución del cromo y la uniformidad del relleno del wet blue.

Neutran BMR: Óxido de magnesio, permite una fijación lenta del cromo evitando variaciones significativas de concentraciones de cromo.

Esta tabla fue tomada de nuestro trabajo publicado en la Revista Sociedad Química del Perú 2013;

79(4):388-397.

\section{Caracterización de los efluentes en los procesos evaluados}

La concentración del cromo total en los efluentes del proceso de curtido fue medido por el método de oxidación alcalina de hipobromito (método HACH 8024) , $^{10}$ equivalente al SM 3500-Cr B (método colorimétrico de la 1,5-difenilcarbazida). En este método el cromo trivalente presente en la muestra se oxida a la forma hexavalente por el ion hipobromito bajo condiciones alcalinas; luego la muestra es acidificada y, finalmente, el contenido total de cromo se determina por el método 1,5 Diphenyl carbohydrazide. Este método tiene una precisión del $95 \%$, mientras que su sensibilidad es $0,005 \mathrm{mg} / \mathrm{L}$; se debe mencionar que antes de iniciar el ensayo es necesario un $\mathrm{pH}$ alrededor de 4 . La lectura se realiza a $540 \mathrm{~nm}$. La determinación del porcentaje de proteínas fue medida por el método SM 4500-Norg C ${ }^{11}$.

\section{Tratamiento de los efluentes por precipitación}

La remoción del cromo en los efluentes de los tratamientos evaluados fue realizada por precipitación química con diferentes dosis de hidróxido de sodio ${ }^{2}$. Se determinó la influencia del pH sobre la precipitación.

\section{Análisis estadístico de los resultados}

El software utilizado fue IBM SPSS Statistics 19. La validación estadística de los datos fue evaluada por ANOVA (Anlysis of Varience); para analizar si los tratamientos influyen en los parámetros analizados; la Prueba de Tukey permite evaluar la significancia entre los 
tratamientos. Para ambos casos el nivel de significancia fue del 5\%. Se planteó un diseño completo, al azar, para los tratamientos de los cinco procesos desarrollados; para cada tratamiento hubo dos repeticiones, considerando a cada tratamiento como unidad experimental.

\section{Influencia del uso de agentes acomplejantes y basificantes sobre el tratamiento del efluente}

Se determinó la influencia del uso de acomplejantes y basificantes sobre la dosis de hidróxido de sodio utilizada en el tratamiento mediante el uso del coeficiente de correlación lineal de Pearson $^{12}$.

\section{Evaluación económica del tratamiento}

Se realizó una internalización de las externalidades del tratamiento sobre el costo de producción de cueros representado en una curva de costo de producción y el costo de tratamiento del efluente ${ }^{9}$.

\section{RESULTADOS Y DISCUSIÓN}

\section{Caracterización del efluente final}

Como se observa en la tabla 2, los efluentes generados por los procesos de curtido T, AI, AII, AIII y AIV, presentan concentraciones finales de cromo total que varía entre 2000 y $100 \mathrm{mg} / \mathrm{L}$, siendo el proceso tradicional el de mayor concentración final; esto es debido a que en este tratamiento se utilizó la mayor dosis de cromo en el proceso; también se observa que el porcentaje de proteínas en el efluente varía entre 2,76 y 3,78\%; esta variabilidad depende del tipo de piel utilizada en el proceso de curtido.

La diferencia entre los volúmenes del proceso tradicional y alternativo I se debe al agua que suelta la piel durante la acción rotativa del botal. El contenido de proteínas en el efluente es importante ya que este influye en la precipitación química, dado que a mayor contenido proteico en el efluente se generará mayores reacciones de peptización.

Tabla 2. Caracterización del efluente de los diferentes procesos de curtido

\begin{tabular}{lccccccc}
\hline Proceso & $\begin{array}{c}\text { Masa de } \\
\text { cromo 33 } \\
25,5 \% \\
(\mathrm{~kg})\end{array}$ & $\begin{array}{c}\text { Volumen } \\
(\mathrm{L})\end{array}$ & $\begin{array}{c}\text { Volumen } \\
\text { final del } \\
\text { baño } \\
(\mathrm{L})\end{array}$ & $\begin{array}{c}\% \text { de } \\
\text { Proteínas } \\
\text { inicial }\end{array}$ & $\begin{array}{c}\text { Cromo } \\
\text { total } \\
\text { inicial } \\
(\mathrm{mg} / \mathrm{L})\end{array}$ & $\begin{array}{c}\% \text { de } \\
\text { Proteínas } \\
\text { final }\end{array}$ & $\begin{array}{c}\text { Cromo } \\
\text { total } \\
\text { final } \\
(\mathrm{mg} / \mathrm{L})\end{array}$ \\
\hline $\mathrm{T}$ & 19,200 & 200,00 & 270,0 & 4,87 & 6500 & 2,76 & 2000,00 \\
$\mathrm{AI}$ & 12,100 & 88,00 & 140,0 & 5,86 & 9310 & 3,74 & 500,00 \\
$\mathrm{AII}$ & 0,115 & 2,10 & 1,5 & 5,70 & 3724 & 3,52 & 650,00 \\
$\mathrm{~A}$ III & 0,485 & 3,88 & 3,0 & 4,17 & 8464 & 3,69 & 200,00 \\
$\mathrm{AIV}$ & 0,147 & 2,94 & 2,0 & 6,02 & 3385 & 3,78 & 100,00 \\
\hline
\end{tabular}

El proceso tradicional (T) y el proceso alternativo I (AI) se desarrollaron a escala industrial; mientras que los demás procesos alternativos (AII, AIII y AIV), se desarrollaron a escala de laboratorio.

La concentración de cromo inicial, es la concentración de cromo que ingresa a la etapa de curtido; mientras que la concentración de cromo final, es la concentración de cromo que sale en el efluente de la etapa. 


\section{Precipitación del cromo}

Como se observa en la tabla 3, la precipitación del cromo como hidróxido de cromo presenta una máxima remoción para dosis entre 0,5 y 5,5 g/L de lentejas de hidróxido de sodio, donde esta dosis depende de la concentración inicial de cromo en el efluente, siendo el tradicional el que requiere mayor dosis; sin embargo, todos los tratamientos presentan concentraciones de cromo total por debajo de los VMA. Como se observa en la figura 1, pasado estas dosis existe una redisolución del cromo por la formación de complejos hidroxilados de cromo III $^{13} \mathrm{y}$ complejos asociados a la proteína en su forma aniónica; esta redisolución por peptización del cromo $^{2}$ puede volver a incrementar valores de cromo total $(250 \mathrm{mg} / \mathrm{L})$ en el efluente filtrado, superando los VMA, como es el caso del proceso $\mathrm{T}$.

Tabla 3. Dosis de hidróxido de sodio para el tratamiento de efluentes de curtido.

\begin{tabular}{lccccccc}
\hline Proceso & $\begin{array}{c}\text { Concentración } \\
\text { inicial de } \\
\mathrm{Cr}\end{array}$ & $\begin{array}{c}\mathrm{pH} \\
\text { inicial } \\
(\mathrm{mg} / \mathrm{L})\end{array}$ & $\begin{array}{c}\text { Dosis } \\
\text { óptima } \\
\text { de NaOH } \\
(\mathrm{g} / \mathrm{L})\end{array}$ & $\begin{array}{c}\text { Concentración } \\
\text { final de } \\
\mathrm{Cr} \\
(\mathrm{mg} / \mathrm{L})\end{array}$ & $\begin{array}{c}\mathrm{pH} \\
\text { final }\end{array}$ & $\begin{array}{c}\mathrm{VMA}^{*} \\
\mathrm{Cr} \\
(\mathrm{mg} / \mathrm{L})\end{array}$ & $\begin{array}{c}\mathrm{VMA}^{*} \\
\mathrm{pH}\end{array}$ \\
\hline $\mathrm{T}$ & 2000 & 3,10 & 5,5 & 2,50 & 10,71 & & \\
$\mathrm{AI}$ & 500 & 4,43 & 3,0 & 0,01 & 9,35 & & \\
AII & 650 & 4,39 & 2,5 & 0,11 & 9,16 & 10 & $6-9$ \\
AIII & 200 & 4,35 & 0,6 & 0,01 & 7,36 & & \\
AIV & 200 & 4,69 & 0,5 & 0,01 & 6,76 & & \\
\hline \hline
\end{tabular}

(*): DS N 021-2009-VIVIENDA. Aprueban Valores Máximos Admisibles (VMA) de las descargas de aguas residuales no domésticas en el sistema de alcantarillado sanitario.

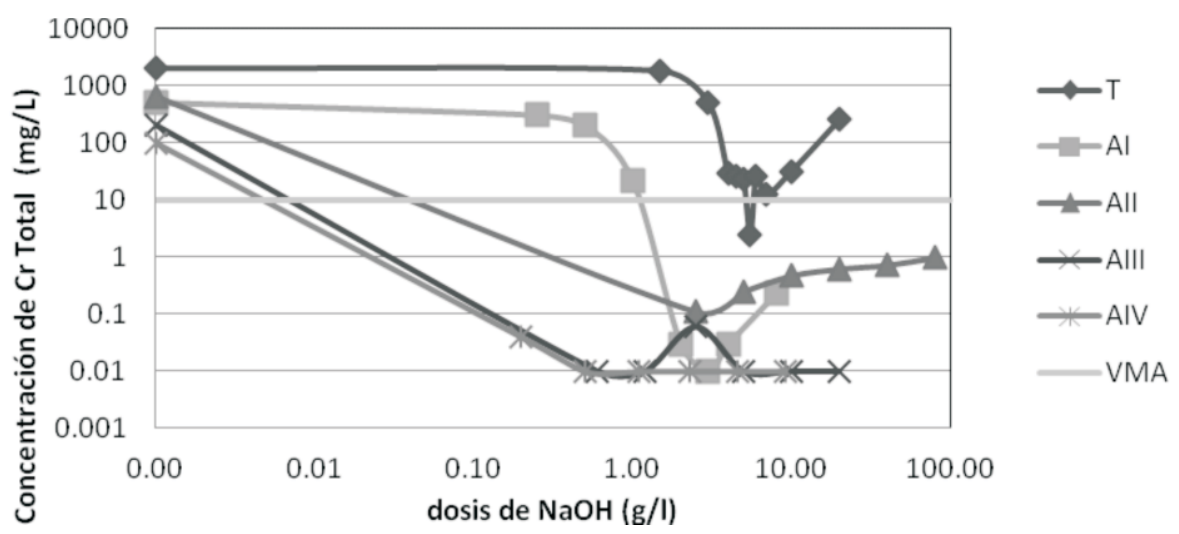

Figura 1. Variación de la concentración de cromo total en el efluente tratado 


\section{Influencia del pH en el tratamiento}

Como se observa en la figura 2, la cantidad del parámetro cromo total disminuye en los efluentes de los cinco procesos de curtido, cuando se incrementa el valor del $\mathrm{pH}$, alcanzando valores mínimos a $\mathrm{pH}$ entre 7,36 y 9,99, similar a lo propuesto por Abass et. al²; la forma como se remueve el cromo del efluente es como hidróxido de cromo. A valores de $\mathrm{pH}$ inferiores a 7,36 o superiores a 9,99 se produce la redisolución del hidróxido de cromo por peptización, que consiste en la redispersión de una sustancia recién precipitada, por efecto de un ion común con el precipitado; en este caso exceso de iones hidroxilo y proteína en su forma aniónica.

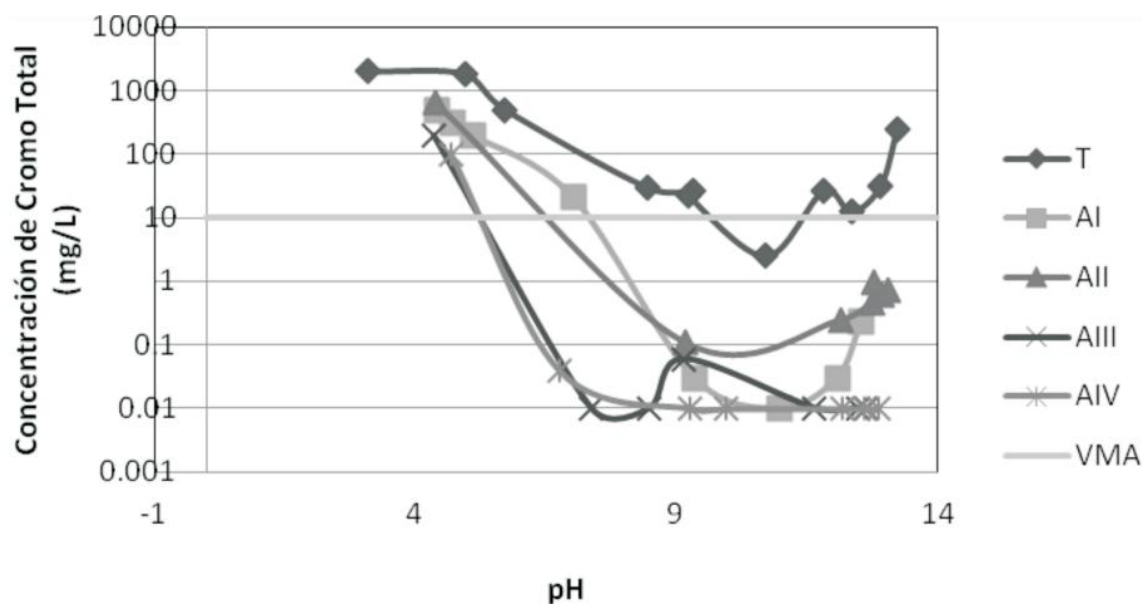

Figura 2. Variación de la concentración de cromo en función del pH del efluente

\section{Análisis estadístico de los resultados}

Para el caso del cromo total en los procesos desarrollados, según el análisis de ANOVA al menos un proceso influye sobre la concentración de cromo (el valor de Significancia es menor a 0,05 por lo que se rechaza la hipótesis de igualdad de varianzas) ${ }^{12}$. Según el análisis de Tukey, los procesos alternativos I, II, III y IV no tienen diferencia significativa, por lo que estadísticamente son similares. A diferencia de los procesos mencionados, el proceso tradicional presenta diferencia significativa ante los demás procesos desarrollados, básicamente por la concentración inicial del cromo (2000 mg/L) logrando una concentración promedio final de $2,5 \mathrm{mg} / \mathrm{L}$. En cambio, para los procesos alternativos I, II, III y IV se inician con $500 \mathrm{mg} / \mathrm{L}, 650 \mathrm{mg} / \mathrm{L}, 200 \mathrm{mg} / \mathrm{L}$ y $100 \mathrm{mg} / \mathrm{L}$, respectivamente.

Como se observa en la figura 3 , en el diagrama de cajas, el tratamiento del efluente del proceso T difiere significativamente con los tratamientos de los efluentes de los procesos alternativos. 


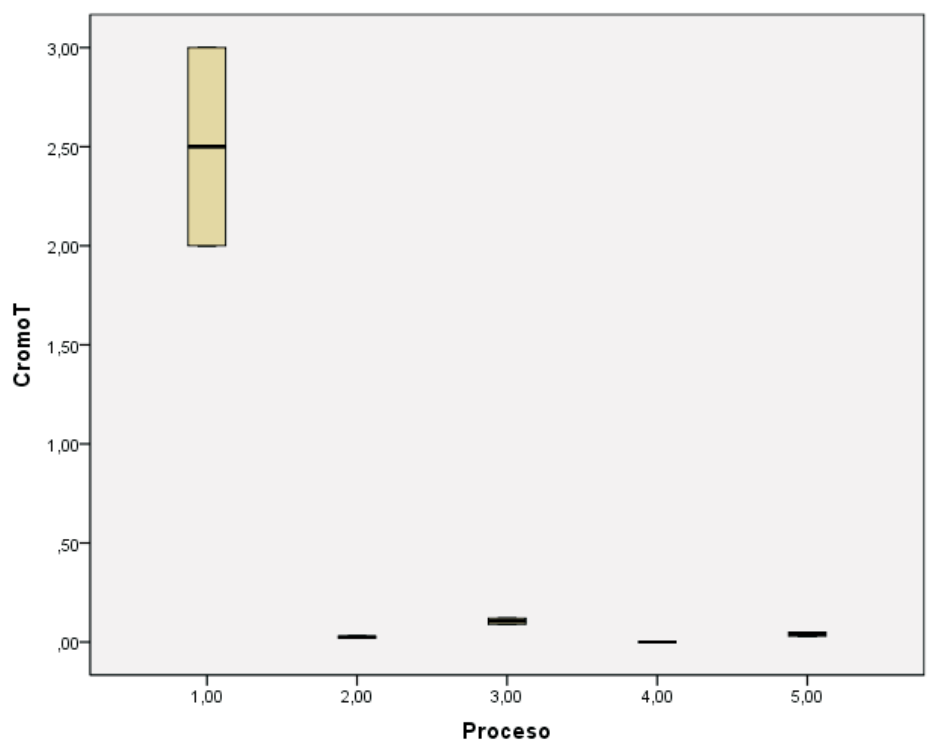

Figura 3. Diagrama de cajas del tratamiento de los efluentes de los procesos tradicional y alternativos.

\section{Influencia del uso de agentes acomplejantes y basificantes sobre el tratamiento del efluente}

El uso de acomplejantes y basificantes de cromo en el proceso de curtido mejora la calidad del efluente con respecto al parámetro cromo; esto debido que el uso de estos insumos permite una mejor distribución del cromo en la piel y mejora el agotamiento del cromo en el baño obteniendo una menor concentración de cromo total en el efluente. Por tal motivo, los procesos en los cuales se usó agentes acomplejantes y basificantes de cromo tienen mayor tratabilidad que un proceso tradicional.

La dosis de hidróxido de sodio utilizada en el tratamiento de los efluentes de los diferentes procesos evaluados tiene un muy buen coeficiente de correlación positiva con la concentración inicial de cromo total en el efluente $(\mathrm{p}=0,98)$; con los agentes acomplejantes Tamplex FLA, Tamplex SS y el Tamplex CRII tiene una muy buena correlación negativa ( $\mathrm{p}=$ $-0,89,-0,84,-0,81)$; finalmente, con el agente basificante Neutran BMR no tiene buena correlación $(\mathrm{p}=-0,5)$.

\section{Evaluación económica del tratamiento}

Debido a que el proceso alternativo II, III y IV se desarrollaron a escala de laboratorio, la evaluación económica del uso de complejantes y basificantes de cromo en el proceso de curtido se realizó en comparación al proceso tradicional y el proceso alternativo I que se desarrollaron a escala industrial. En esta evaluación se compara el costo de los insumos de producir en nuevos soles un pie $^{2}$ de cuero al estado wet blue con el costo de los insumos para tratar los efluentes. 
Como se observa en la figura 4 , el tratamiento de efluentes de curtido con valores menores a $650 \mathrm{mg} / \mathrm{L}$ de cromo total, el costo de los insumos empleados en la producción del cuero a wet blue es mayor al costo de insumos empleados en el tratamiento. Por el contrario, para efluentes con valores mayores el costo de los insumos de tratamiento son mucho mayores que los costos en insumos de producción. Se debe considerar las externalidades, gastos o beneficios no controlados, del tratamiento en el costo del producto final cuero wet blue.

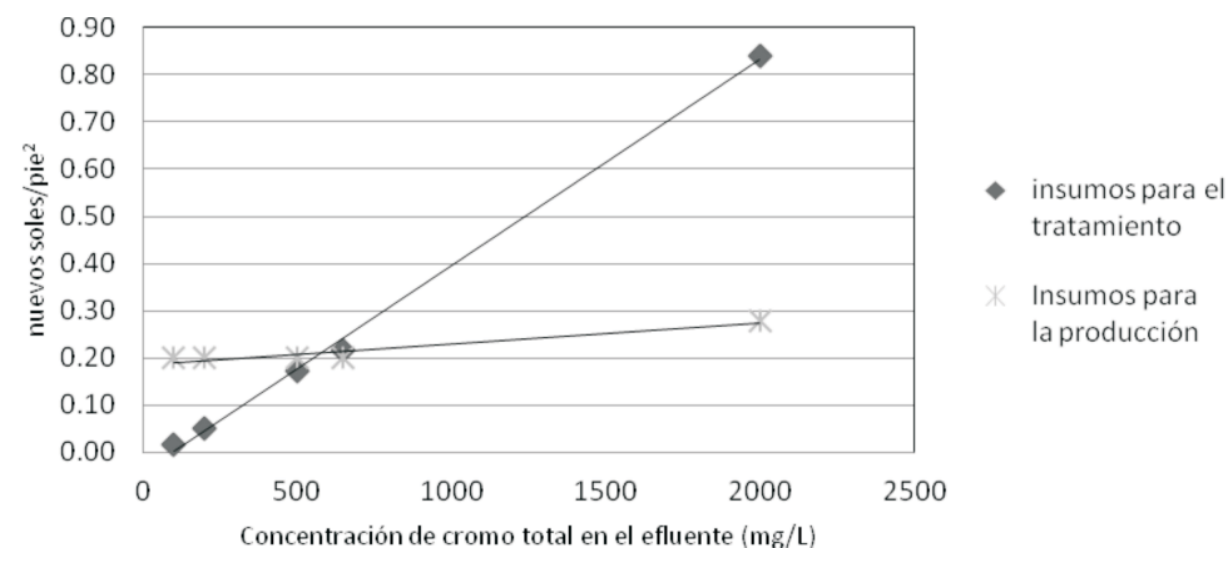

Figura 4. Comparación de costos de insumos de producción con insumos para el tratamiento

\section{CONCLUSIONES}

El proceso tradicional requiere de mayor dosis de hidróxido de sodio $(5 \mathrm{~g} / \mathrm{L})$ para la precipitación del cromo, comparado con los procesos alternativos usando complejantes de cromo $(0,5-3,5 \mathrm{~g} / \mathrm{L})$. La precipitación del cromo total del efluente se lleva a valores de $\mathrm{pH}$ entre 7,36 y 9,99. La dosis de hidróxido de sodio utilizada en el tratamiento de los efluentes de los diferentes procesos evaluados tiene una muy buena correlación con los agentes acomplejantes Tamplex FLA, Tamplex SS y el Tamplex CRII. Sin embargo, el agente basificante Neutran BMR no tiene buena correlación con la dosis de hidróxido de sodio.

El costo de los insumos usados en el tratamiento del efluente de curtido es mayor en relación al costo de los insumos usados en el proceso de producción del cuero cuando la cantidad de cromo total del efluente sobrepasa los $650 \mathrm{mg} / \mathrm{L}$.

\section{AGRADECIMIENTOS}

Se agradece al Fondo de Investigación y Desarrollo para la Competitividad (FIDECOM) que a través de los Proyectos de Innovación Productiva para Empresas Individuales (PIPEI) por el Convenio $\mathrm{N}^{\circ}$ 022-FINCYT-FIDECOM-PIPEI-2012 entre la empresa HELIANTHUS S.A.C y el Centro de Investigación en Química, Toxicología y Biotecnología Ambiental (CIQTOBIA) del Departamento Académico de Química de la UNALM financiaron la investigación. 


\section{BIBLIOGRAFÍA}

1. Helianthus SAC. (2013), Ranking de Ventas por Cliente, Sistema SOINFO del 09 de octubre 2012 al 09 de Octubre 2013, Lima - Perú.

2. Abass Esmaeili, Alireza Mesdaghi nia and Reza Vazirinejad. Chromium (III) Removal and Recovery from Tannery Wastewater by Precipitation Process. American Journal of Applied Sciences, 2005; 2 (10): 1471-1473.

3. Barsoum, B., Eid, A., Fahim, N., \& Khalil, M. Removal of chromium (III) from tannery wastewater using activated carbon from sugar industrial waste. Journal of Hazardous Materials, 2006: 303-309.

4. Boaventura, R., \& Fonseca, A. Chromium precipitation from tanning spent liquors using industrial alkaline residues: A comparative study. Portugal: University of Porto, 1997. Waste Management, 1997; 17(4): 201-209.

5. Patterson, J.W., Industrial wastewater treatment technology: Butter Worths: Boston, 1985.

6. Kocaoba, S. and G. Akin. Removal and recovery of chromium and chromium speciation with MINTEQA2. Talanta, 2002; 57(1):23-30.

7. Tsugita, R.A. and R.H, Ellis. Pretreatment of industrial wastes manual of practice. WPCF No ED-3, 1981.

8. United Nations Industrial Development Organization UNIDO, Chrome Management in the Tanyard, US/RAS/92/120/11-51 2000

9. Juan Martínez Alier, Curso de Economía Ecológica, Programa de las Naciones Unidas para el Medio Ambiente Oficina Regional para América Latina y el Caribe, 1998: 53 - 55.

10. HACH, Alkaline Hypobromite Oxidation Method (Method HACH 8024), Adaptado del Standard Method 3500-Cr D for wastewater 2010.

11. Standard Methods for the Examination of Water and Wastewater 20 $0^{\text {th }}$ Edition 1998. Parte 4500-Norg C. Método Semimicro Kejdahl.

12. Salkind, N. Métodos de investigación. Prentice Hall. México, 1999:225-226.

13 Ávila, M., Gonzales, M., Navarro, R., Saucedo, R. \& Turriza, R. Ultrafiltración asistida con formación de complejos para la recuperación de cromo (III) de soluciones acuosas. VIII Simposio Internacional de Qualidade Ambiental. 2012. 